\title{
On Circuits and Pancyclic Line Graphs
}

A. Benhocine UNIVERSITE DU MAINE

LE MANS, FRANCE

L. Clark

UNIVERSITY OF NEW MEXICO

ALBUQUERQUE, NM

N. Köhler

GERLINGERSTRASSE 6 .

D-1000 BERLIN 47

H. J. Veldman

TWENTE UNIVERSITY OF TECHNOLOGY

ENSCHEDE, THE NETHERLANDS

\section{ABSTRACT}

Clark proved that $L(G)$ is hamiltonian if $G$ is a connected graph of order $n \geq 6$ such that deg $u+\operatorname{deg} v \geq n-1-p(n)$ for every edge $u v$ of $G$, where $p(n)=0$ if $n$ is even and $p(n)=1$ if $n$ is odd. Here it is shown that the bound $n-1-p(n)$ can be decreased to $(2 n+1) / 3$ if every bridge of $G$ is incident with a vertex of degree 1 , which is a necessary condition for hamiltonicity of $L(G)$. Moreover, the conclusion that $L(G)$ is hamiltonian can be strengthened to the conclusion that $L(G)$ is pancyclic. Lesniak-Foster and Williamson proved that $G$ contains a spanning closed trail if $|V(G)|=n \geq 6, \delta(G) \geq 2$ and deg $u+\operatorname{deg} v \geq n-1$ for every pair of nonadjacent vertices $u$ and $v$. The bound $n-1$ can be decreased to $(2 n+3) / 3$ if $G$ is connected and bridgeless, which is necessary for $G$ to have a spanning closed trail.

\section{TERMINOLOGY}

We use [4] for basic terminology and notation, but speak of vertices and edges instead of points and lines. Accordingly we denote the edge set of a graph $G$ by $E(G)$. In [7] a circuit was defined as a nontrivial closed trail. Here the following subtle variation on this definition will be more convenient. A circuit $C$ of a graph $G$ is a nontrivial eulerian subgraph of $G$. Alternatively, $C$ is a circuit if 
and only if $C$ is a nontrivial connected subgraph such that every vertex of $C$ has even degree in $C$. If $C$ is a circuit of $G$, then $\beta(C)$ denotes the number of edges of $G$ incident with at least one vertex of $C$. A spanning circuit, or briefly $S$-circuit, of a graph $G$ is a circuit that contains all vertices of $G$. A dominating circuit or $D$-circuit of $G$ is a circuit such that every edge of $G$ is incident with at least one vertex of the circuit. If $H$ is a subgraph of $G$, then vertices of $G-V(H)$ which are adjacent to at least one vertex of $H$ are called neighbors of $H$. We denote the neighbors of $H=\{v\}$ by $N\{v\}$. A graph of order $n$ is pancyclic if it contains a cycle of length $i$ for each $i$ with $3 \leq i \leq n$. A chord of a cycle $C$ in $G$ is an edge in $E(G)-E(C)$ whose ends are in $C$. A connected graph $G$ is said to be almost bridgeless if every bridge of $G$ is incident with a vertex of degree 1. If $x$ is a real number, then $\lfloor x\rfloor$ and $\lceil x\rceil$ denote, respectively, the greatest integer smaller than or equal to $x$ and the smallest integer greater than or equal to $x$.

\section{DOMINATING CIRCUITS AND PANCYCLIC LINE GRAPHS}

In [5] the following relation between $D$-circuits in graphs and hamiltonian cycles in line graphs is established.

Theorem 1. (Harary and Nash-Williams [5]). The line graph $L(G)$ of a graph $G$ contains a hamiltonian cycle if and only if $G$ has a $D$-circuit or $G$ is isomorphic to $K_{1, s}$ for some $s \geq 3$.

In [3] Clark proved that the line graph $L(G)$ of a graph $G$ is hamiltonian if $G$ is connected, $|V(G)|=n \geq 6$ and $\operatorname{deg} u+\operatorname{deg} v \geq n-1-p(n)$ for every edge $u v$ of $G$, where $p(n)=0$ if $n$ is even and $p(n)=1$ if $n$ is odd. The graphs showing that Clark's result is best possible all contain a bridge which is not incident with a vertex of degree 1 . If a graph $G$ contains a bridge $u v$ with $\operatorname{deg} u \neq 1 \neq \operatorname{deg} v$, then the vertex of $L(G)$ corresponding to $u v$ is a cut vertex of $L(G)$, so that $L(G)$ is nonhamiltonian. Hence a necessary condition for $L(G)$ to have a hamiltonian cycle, and for $G$ to have a $D$-circuit, is that $G$ is almost bridgeless. Using Theorem 1 we will show how Clark's bound $n-1$ $p(n)$ can be decreased if $G$ is additionally required to be almost bridgeless. Before presenting our result we state two lemmas, the first of which is easily proved and frequently used in [2] and [3].

Lemma 2. Let $G$ be a connected graph and $C$ a circuit of $G$ with maximum number of vertices. Then $G$ contains no circuit $C^{\prime}$ satisfying $V\left(C^{\prime}\right) \cap V(C) \neq$ $\emptyset \neq V\left(C^{\prime}\right) \cap V(G)-V(C)$ and $\left|E\left(C^{\prime}\right) \cap E(C)\right| \leq 1$.

Lemma 3. Let $G$ be a connected graph, $C$ a circuit of $G$ with maximum number of vertices, $K$ a component of $G-V(C)$ and $u_{1}$ and $u_{2}$ two neighbors of $K$ on $C$. Then the following assertions hold. 
a. $u_{1}$ and $u_{2}$ are nonadjacent.

b. If $w \in N\left(u_{1}\right) \cap N\left(u_{2}\right)-V(K)$, then none of the vertex pairs $\left\{u_{1}, w\right\}$ and $\left\{u_{2}, w\right\}$ has a common neighbor.

c. If $w_{1} \in N\left(u_{1}\right)-V(K), w_{2} \in N\left(u_{2}\right)-V(K)$ and $w_{1} w_{2} \in E(G)$, then at most one of the pairs $\left\{u_{1}, w_{1}\right\},\left\{u_{2}, w_{2}\right\}$, and $\left\{w_{1}, w_{2}\right\}$ has a common neighbor.

d. If $v \in V(K)$ and $w \in N\left(u_{1}\right) \cap N\left(u_{2}\right)-V(K)$, then $v$ and $w$ are nonadjacent and have no common neighbor in $G-\left(V(K) \cup\left\{u_{1}, u_{2}\right\}\right)$.

e. If $w_{1}, w_{2} \in N\left(u_{1}\right) \cap N\left(u_{2}\right)-V(K)$, then $w_{1}$ and $w_{2}$ are nonadjacent and have no common neighbor in $G-\left\{u_{1}, u_{2}\right\}$.

Proof. Let $G$ be a connected graph, $C$ a circuit of $G$ of maximum order, $K$ a component of $G-V(C)$ and $u_{1}$ and $u_{2}$ two neighbors of $K$ on $C$. Throughout the proof $P$ will denote a $u_{1}-u_{2}$ path with $\emptyset \neq V(P)-\left\{u_{1}, u_{2}\right\} \subset V(K)$.

a. Suppose $u_{1} u_{2} \in E(G)$. Then the cycle with edge set $E(P) \cup\left(u_{1} u_{2}\right\}$ contradicts the assertion of Lemma 2. Hence $u_{1}$ and $u_{2}$ are nonadjacent.

b. Let $w$ be a vertex of $N\left(u_{1}\right) \cap N\left(u_{2}\right)-V(K)$. If $u_{1} w \notin E(C)$ or $u_{2} w \notin$ $E(C)$ then the cycle with edge set $E(P) \cup\left\{u_{1} w, u_{2} w\right\}$ contradicts Lemma 2. Hence $u_{1} w, u_{2} w \in E(C)$. Suppose, for example, $u_{1}$ and $w$ have a common neighbor $v$. From Lemma 2 we deduce that $v \in V(C)$ and at least one of the edges $u_{1} v$ and $v w$ is in $E(C)$. Depending on whether or not each of the edges $u_{1} v$ and $v w$ is in $E(C)$ we now define a subgraph $C^{\prime}$ of $G$ by specifying $E\left(C^{\prime}\right)-E(C)$ and $E(C)-E\left(C^{\prime}\right) ; V\left(C^{\prime}\right)$ will be the set of vertices of $G$ incident with at least one edge of $E\left(C^{\prime}\right)$. In the table below there is a column for each of the edges $u_{1} v$ and $v w$; a one in such a column means that the relevant edge is in $E(C)$, while a zero means that it is in $E(G)-E(C)$.

$$
\begin{array}{ccll}
\frac{u, v}{1} & \frac{v w}{1} & \frac{E\left(C^{\prime}\right)-E(C)}{E(P)} & \frac{E(C)-E\left(C^{\prime}\right)}{\left\{u_{1} w, u_{2} w\right\}} \\
1 & 0 & E(P) \cup\{v w\} & \left\{u_{1} v, u_{2} w\right\} \\
0 & 1 & E(P) \cup\left\{u_{1} v\right\} & \left\{v w, u_{2} w\right\}
\end{array}
$$

If, for example, $u_{1} v \in E(C)$ and $v w \notin E(C)$, then $C^{\prime}$ is defined as the subgraph of $G$ with $V\left(C^{\prime}\right)=V(C) \cup V(P)$ and $E\left(C^{\prime}\right)=E(C) \cup E(P) \cup$ $\{v w\}-\left\{u_{1} v, u_{2} w\right\}$, as indicated in the second row of the table. In all cases the fact that $C$ is connected implies that $C^{\prime}$ is connected. Furthermore, since all vertices of $C$ have even degree in $C$, all vertices of $C^{\prime}$ have even degree in $C^{\prime}$. It follows that $C^{\prime}$ is a circuit with $\left|V\left(C^{\prime}\right)\right|=$ $|V(C) \cup V(P)|>|V(C)|$, contradicting the choice of $C$ and completing the proof of (b).

c. Let $w_{1}$ and $w_{2}$ be vertices of $G$ such that $w_{1} \in N\left(u_{1}\right)-V(K), w_{2} \in$ $N\left(u_{2}\right)-V(K)$ and $w_{1} w_{2} \in E(G)$. By Lemma 2 at least two of the edges $u_{1} w_{1}, w_{1} w_{2}$ and $u_{2} w_{2}$ are in $E(C)$. If one of the three edges is in $E(G)-$ 
$E(C)$, then a slight variation on the arguments used in (a) yields that the vertices incident with each of the remaining edges have no common neighbor. Hence assume $u_{1} w_{1}, w_{1} w_{2}, u_{2} w_{2} \in E(C)$. Suppose that at least two of the pairs $\left\{u_{1}, w_{1}\right\},\left\{w_{1}, w_{2}\right\}$ and $\left\{u_{2}, w_{2}\right\}$ have a common neighbor. We derive contradictions in two cases.

Case 1. There exists a vertex $w$ of $G$ which is adjacent to at least three of the vertices $u_{1}, u_{2}, w_{1}, w_{2}$.

From Lemma 2 and (b) we deduce that $w \in V(C)-\left\{u_{1}, u_{2}, w_{1}, w_{2}\right\}$ and $w$ is adjacent to $w_{1}, w_{2}$ and exactly one of the vertices $u_{1}$ and $u_{2}, u_{1}$ say. Lemma 2 also implies that at least one of the edges $w u_{1}$ and $w w_{2}$ is in $E(C)$. In all possible cases we now specify, like in the proof of (b), a circuit $C^{\prime}$ of $G$ with $\left|V\left(C^{\prime}\right)\right|>|V(C)|$, contradicting the choice of $C$.

$\begin{array}{cccll}\frac{w u_{1}}{1} & \frac{w w_{1}}{1} & \frac{w w_{2}}{1} & \frac{E\left(C^{\prime}\right)-E(C)}{E(P)} & \frac{E(C)-E\left(C^{\prime}\right)}{\left\{u_{1} w_{1}, u_{2} w_{2}, w_{1} w_{2}\right\}} \\ 1 & 1 & 0 & E(P) \cup\left\{w w_{2}\right\} & \left\{w u_{1}, u_{2} w_{2}\right\} \\ 1 & 0 & 1 & E(P) \cup\left\{w w_{1}\right\} & \left\{w u_{1}, w_{1} w_{2}, u_{2} w_{2}\right\} \\ 0 & 1 & 1 & E(P) \cup\left\{w u_{1}\right\} & \left\{w w_{2}, u_{2} w_{2}\right\} \\ 1 & 0 & 0 & E(P) \cup\left\{w w_{2}\right\} & \left\{w u_{1}, u_{2} w_{2}\right\} \\ 0 & 0 & 1 & E(P) \cup\left\{w u_{1}\right\} & \left\{w w_{2}, u_{2} w_{2}\right\}\end{array}$

Case 2. Each vertex of $G$ is adjacent to at most two of the vertices $u_{1}, u_{2}$, $w_{1}, w_{2}$.

We assume that $u_{i}$ and $w_{i}$ have a common neighbor $v_{i}(i=1,2)$; the remaining subcases are similar. From Lemma 2 we deduce that $v_{1}, v_{2} \in V(C)$ and at least one of the edges $u_{1} v_{1}, v_{1} w_{1}, u_{2} v_{2}$ and $v_{2} w_{2}$ is in $E(C)$. Again a circuit $C^{\prime}$ of $G$ with $\left|V\left(C^{\prime}\right)\right|>|V(C)|$ can be specified in all possible cases. We only treat two representative cases.

$$
\begin{array}{ccccll}
\frac{u_{1} v_{1}}{1} & \frac{v_{1} w_{1}}{1} & \frac{u_{2} v_{2}}{1} & \frac{v_{2} w_{2}}{0} & \frac{E\left(C^{\prime}\right)-E(C)}{E(P) \cup\left\{v_{2} w_{2}\right\}} & \frac{E(C)-E\left(C^{\prime}\right)}{\left\{u_{1} w_{1}, w_{1} w_{2}, u_{2} v_{2}\right\}} \\
0 & 0 & 0 & 1 & E(P) \cup\left\{u_{1} v_{1}, v_{1} w_{1}, u_{2} v_{2}\right\} & \left\{w_{1} w_{2}, v_{2} w_{2}\right\}
\end{array}
$$

d. Let $v$ be a vertex of $K$ and $w$ a vertex in $N\left(u_{1}\right) \cap N\left(u_{2}\right)-V(K)$. For $i=$ 1,2 , let $P_{i}$ be a $v-u_{i}$ path with all internal vertices in $K$. From Lemma 2 it follows that $v w \notin E(G)$ and $u_{1} w, u_{2} w \in E(C)$. Suppose $v$ and $w$ have a common neighbor $u$ in $G-\left(V(K) \cup\left\{u_{1}, u_{2}\right\}\right)$. Then $u w \in E(C)$ by Lemma 2. If $w$ is not a cut vertex of $C$ or if $u_{1}, u_{2}$ and $u$ are in the same component of $C-w$, then the subgraph $C^{\prime}$ of $G$ with $V\left(C^{\prime}\right)=V(C) \cup$ $V\left(P_{1}\right)$ and $E\left(C^{\prime}\right)=E(C) \cup E\left(P_{1}\right) \cup\{u v\}-\left\{u w, u_{1} w\right\}$ is connected, implying that $C^{\prime}$ is a circuit of $G$ with $\left|V\left(C^{\prime}\right)\right|>|V(C)|$. Hence assume that $w$ is a cut vertex of $C$ and, for example, $u$ and $u_{2}$ are in different components $H_{1}$ and $H_{2}$ of $C-w$, respectively. Let $C_{i}$ be the subgraph of $C$ 
induced by $V\left(H_{i}\right) \cup\{w\}(i=1,2)$. Then $C_{1}$ and $C_{2}$ are subcircuits of $C$. In particular, $C_{1}$ and $C_{2}$ are bridgeless, so $C_{1}-u w$ and $C_{2}-u_{2} w$ are connected subgraphs of $C$. It follows that $C-\left\{u w, u_{2} w\right\}$ is connected. But then the circuit $C^{\prime}$ with $V\left(C^{\prime}\right)=V(C) \cup V\left(P_{2}\right)$ and $E\left(C^{\prime}\right)=E(C) \cup$ $E\left(P_{2}\right) \cup\{u v\}-\left\{u w, u_{2} w\right\}$ contradicts the choice of $C$.

e. Let $w_{1}$ and $w_{2}$ be two vertices in $N\left(u_{1}\right) \cup N\left(u_{2}\right)-V(K)$. Then $u_{i} w_{j} \in$ $E(C)$ by Lemma $2(i=1,2 ; j=1,2)$. The table below shows that a circuit $C^{\prime}$ with $\left|V\left(C^{\prime}\right)\right|>|V(C)|$ can be constructed if $w_{1} w_{2} \in E(G)$.

$\begin{array}{cll}\frac{w_{1} w_{2}}{1} & \frac{E\left(C^{\prime}\right)-E(C)}{E(P)} & \frac{E(C)-E\left(C^{\prime}\right)}{\left\{u_{1} w_{1}, u_{2} w_{1}\right\}} \\ 0 & E(P) \cup\left\{w_{1} w_{2}\right\} & {\left[u_{1} w_{1}, u_{2} w_{2}\right\}}\end{array}$

Suppose $w_{1}$ and $w_{2}$ have a common neighbor $v$ in $G-\left\{u_{1}, u_{2}\right\}$. Again a circuit $C^{\prime}$ with $\left|V\left(C^{\prime}\right)\right|>|V(C)|$ can be specified. Note that in the fourth row of the table below $v$ may be a vertex of $P$.

$\begin{array}{ccll}\frac{v w_{1}}{1} & \frac{v w_{2}}{1} & \frac{E\left(C^{\prime}\right)-E(C)}{E(P)} & \frac{E(C)-E\left(C^{\prime}\right)}{} \\ 1 & 0 & E(P) \cup\left\{v w_{2}\right\} & \left\{u_{1} w_{1}, u_{2} w_{1}\right\} \\ 0 & 1 & E(P) \cup\left\{v w_{1}\right\} & \left\{u_{1} w_{1}, v w_{1}, u_{2} w_{2}\right\} \\ 0 & 0 & E(P) \cup\left\{v w_{1}, v w_{2}\right\} & \left\{u_{1} w_{1}, v w_{2}, u_{2} w_{2}\right\} \\ & 0 & \left.u_{1} w_{1}, u_{2} w_{2}\right\}\end{array}$

Theorem 4. Let $G$ be a nontrivial connected, almost bridgeless graph of order $n$ with $G \not K_{1, n-1}$. If $\operatorname{deg} u+\operatorname{deg} v \geq(2 n+1) / 3$ for every edge $u v$ of $G$, then $G$ contains a $D$-circuit.

Proof. Let $G$ be a connected, almost bridgeless graph of order $n$ with $G \not \equiv$ $K_{1, n-1}$. Assuming that $G$ contains no $D$-circuit, we will exhibit two adjacent vertices with degree-sum at most $\frac{2}{3} n$. Since $G$ is almost bridgeless and $G \not$ $K_{1, n-1}$, deletion of all vertices of degree 1 yields a nontrivial bridgeless graph, implying that $G$ contains a circuit. Let $C$ be a circuit of $G$ such that $|\mathrm{V}(C)|$ is maximum and $\beta(C) \geq \beta\left(C^{\prime}\right)$ for every circuit $C^{\prime}$ with $\left|V\left(C^{\prime}\right)\right|=|V(C)|$. Since $C$ is not a $D$-circuit, $G-V(C)$ has a nontrivial component $K$. From Lemma 2 and the fact that $G$ is almost bridgeless we conclude that $K$ has at least two neighbors on $C$. We distinguish three cases.

Case 1. $K$ has two neighbors on $C$ which are joined by a path of length 2 contained in $G-V(K)$.

Let $u_{1}$ and $u_{2}$ be two neighbors of $K$ on $C$ which are joined by the path $u_{1} w_{1} u_{2}$, where $w_{1} \notin V(K)$. Let $P$ be a $u_{1}-w_{2}$ path with $\emptyset \neq V(P)-$ $\left\{u_{1}, u_{2}\right\} \subset V(K)$ such that $|V(P)|$ is minimum. Define $v_{1}$ as the immediate successor of $u_{1}$ on $P$. If $V(P)-\left\{u_{1}, u_{2}\right\}=\left\{v_{1}\right\}$, let $v_{2}$ be an arbitrary neighbor of $v_{1}$ in $K$, otherwise let $v_{2}$ be the successor of $v_{1}$ on $P$. Finally, let $H$ be the 
induced subgraph $\left.\left\langle V(P) \cup v_{2}, w_{1}\right\}\right\rangle$ of $G$. From Lemmas 2, 3(b) and 3(d) it follows that

$$
\begin{array}{r}
N\left(u_{1}\right) \cap N\left(v_{1}\right) \cap(V(G)-V(H))=N\left(u_{1}\right) \cap N\left(w_{1}\right) \cap(V(G)-V(H)) \\
=N\left(v_{1}\right) \cap N\left(w_{1}\right) \cap(V(G)-V(H))=\emptyset .
\end{array}
$$

We next show that

$$
V(G)-\left(V(H) \cup N\left(u_{1}\right) \cup N\left(\boldsymbol{v}_{1}\right) \cup N\left(w_{1}\right)\right) \neq \emptyset .
$$

Since each vertex of $C$ has even degree in $C, u_{2}$ has a neighbor $w_{2}$ on $C$ with $w_{2} \neq w_{1}$. If $u_{1} w_{2} \notin E(G)$, then, by Lemmas 2 and $3(\mathrm{~b}), w_{2}$ is not adjacent to any of the vertices $u_{1}, v_{1}$ and $w_{1}$, implying (2). Now assume $u_{1} w_{2} \in E(G)$. Then by Lemma 2 we have $u_{1} w_{2}, u_{2} w_{2} \in E(C)$ and $v_{2} w_{2} \notin E(G)$. There exists a vertex $w$ in $G-V(H)$ which is adjacent to $w_{2}$, otherwise the circuit $C^{\prime}$ with $V\left(C^{\prime}\right)=V(C) \cup V(P)-\left\{w_{2}\right\}$ and $E\left(C^{\prime}\right)=E(C) \cup E(P)-\left\{u_{1} w_{2}, u_{2} w_{2}\right\}$ satisfies $\left|V\left(C^{\prime}\right)\right| \geq|V(C)|$ and $\beta\left(C^{\prime}\right)>\beta(C)$, contradicting the choice of $C$. By Lemma 2, $w \notin V(K)$. Application of Lemmas 3(b), 3(d) and 3(e) yields that $w$ is adjacent to none of the vertices $u_{1}, v_{1}$ and $w_{1}$, implying (2).

Equation (1) expresses that each vertex of $G-V(H)$ is adjacent to at most one of the vertices $u_{1}, v_{1}$ and $w_{1}$. Together with (2) we obtain

$$
\begin{aligned}
\operatorname{deg} u_{1}+\operatorname{deg} v_{1}+\operatorname{deg} w_{1} \leq & n-|V(H)|-1+\operatorname{deg}_{H} u_{1}+\operatorname{deg}_{H} v_{1} \\
& +\operatorname{deg}_{H} w_{1} .
\end{aligned}
$$

Similarly,

$$
\begin{aligned}
\operatorname{deg} u_{1}+\operatorname{deg} v_{2}+\operatorname{deg} w_{1} \leq & n-|V(H)|-1+\operatorname{deg}_{H} u_{1}+\operatorname{deg}_{H} v_{2} \\
& +\operatorname{deg}_{H} w_{1} .
\end{aligned}
$$

Summation of the inequalities (3) and (4) yields

$$
\begin{aligned}
& 2\left(\operatorname{deg} u_{1}+\operatorname{deg} w_{1}\right)+\operatorname{deg} v_{1}+\operatorname{deg} v_{2} \\
& \quad \leq 2\left(n-|V(H)|-1+\operatorname{deg}_{H} u_{1}+\operatorname{deg}_{H} w_{1}\right)+\operatorname{deg}_{H} v_{1}+\operatorname{deg}_{H} v_{2} .
\end{aligned}
$$

From Lemma 2, Lemma 3(a) and the minimality of $|V(P)|$ we conclude that every vertex of $H-\left\{v_{1}, v_{2}\right\}$ has degree 2 in $H$. Furthermore, $\operatorname{deg}_{H} v_{1}=$ $\operatorname{deg}_{H} v_{2}=2$ if $v_{2} \in V(P)$, while $\operatorname{deg}_{H} v_{1}=3$ and $\operatorname{deg}_{H} v_{2}=1$ otherwise. Observing that $|V(H)| \geq 5$ we now deduce from (5) that

$$
2\left(\operatorname{deg} u_{1}+\operatorname{deg} w_{1}\right)+\operatorname{deg} v_{1}+\operatorname{deg} v_{2} \leq 2 n .
$$

It follows that either $\operatorname{deg} u_{1}+\operatorname{deg} w_{1} \leq \frac{2}{3} n$ or $\operatorname{deg} v_{1}+\operatorname{deg} v_{2} \leq \frac{2}{3} n$, settling Case 1. 
Case 2. Case 1 does not apply and $K$ has two neighbors on $C$ which are joined by a path of length 3 contained in $G-V(K)$.

Let $u_{1}$ and $u_{2}$ be two neighbors of $K$ on $C$ which are joined by the path $u_{1} w_{1} w_{2} u_{2}$, where $w_{1}, w_{2} \notin V(K)$. Define $P, v_{1}$ and $v_{2}$ as in Case 1 and put $H=\left\langle V(P) \cup\left\{v_{2}, w_{1}, w_{2}\right\}\right\rangle$. By Lemma $3(\mathrm{c})$ at least one of the pairs $\left\{u_{1}, w_{1}\right\}$ and $\left\{u_{2}, w_{2}\right\},\left\{u_{1}, w_{1}\right\}$ say, has no common neighbor. In particular,

$$
N\left(u_{1}\right) \cap N\left(w_{1}\right) \cap(V(G)-V(H))=\emptyset .
$$

By Lemma $2, v_{1}$ and $w_{1}$ have no common neighbor outside $C$. Suppose $v_{1}$ and $w_{1}$ have a common neighbor $u$ on $C$ with $u \neq u_{1}$. Then Case 1 applies to the neighbors $u$ and $u_{1}$ of $K$ on $C$, contrary to assumption. We conclude that

$$
N\left(v_{1}\right) \cap N\left(w_{1}\right) \cap(V(G)-V(H))=\emptyset .
$$

Another application of Lemma 2 gives us

$$
N\left(u_{1}\right) \cap N\left(v_{1}\right) \cap(V(G)-V(H))=\emptyset .
$$

From (6), (7), and (8) we deduce that

$$
\operatorname{deg} u_{1}+\operatorname{deg} v_{1}+\operatorname{deg} w_{1} \leq n-|V(H)|+\operatorname{deg}_{H} u_{1}+\operatorname{deg}_{H} v_{1}+\operatorname{deg}_{H} w_{1} .
$$

Similarly,

$$
\operatorname{deg} u_{1}+\operatorname{deg} v_{2}+\operatorname{deg} w_{1} \leq n-|V(H)|+\operatorname{deg}_{H} u_{1}+\operatorname{deg}_{H} v_{2}+\operatorname{deg}_{H} w_{1} .
$$

Summation of $(9)$ and $(10)$ yields

$$
\begin{aligned}
& 2\left(\operatorname{deg} u_{1}+\operatorname{deg} w_{1}\right)+\operatorname{deg} v_{1}+\operatorname{deg} v_{2} \\
& \quad \leq 2\left(n-|V(H)|+\operatorname{deg}_{H} u_{1}+\operatorname{deg}_{H} w_{1}\right)+\operatorname{deg}_{H} v_{1}+\operatorname{deg}_{H} v_{2} .
\end{aligned}
$$

By Lemmas 2, 3(a), 3(b) and the minimality of $|V(P)|$, every vertex of $H-\left\{v_{1}, v_{2}\right\}$ has degree 2 in $H$, while $\operatorname{deg}_{H} v_{1}+\operatorname{deg}_{H} v_{2}=4$. Observing that $|V(H)| \geq 6$, we deduce from (11) that

$$
2\left(\operatorname{deg} u_{1}+\operatorname{deg} w_{1}\right)+\operatorname{deg} v_{1}+\operatorname{deg} v_{2} \leq 2 n,
$$

implying that either $\operatorname{deg} u_{1}+\operatorname{deg} w_{1} \leq \frac{2}{3} n$ or $\operatorname{deg} v_{1}+\operatorname{deg} v_{2} \leq \frac{2}{3} n$.

Case 3. Neither Case 1 nor Case 2 applies.

Let $u_{1}$ and $u_{2}$ be two arbitrary neighbors of $K$ on $C$ and $w$ a vertex in $N\left(u_{2}\right)-V(K)$. Define $P, v_{1}$ and $v_{2}$ as in Case 1 and put $H=\left\langle V(P) \cup\left\{v_{2}, w\right\}\right\rangle$. 
By Lemma 2 and by assumption we have

$$
\begin{aligned}
N\left(u_{1}\right) \cap N\left(v_{1}\right) \cap(V(G)-V(H)) & =N\left(u_{2}\right) \cap N\left(v_{1}\right) \cap(V(G)-V(H)) \\
& =N\left(u_{1}\right) \cap N\left(u_{2}\right) \cap(V(G)-V(H))=\emptyset,
\end{aligned}
$$

implying that

$$
\begin{aligned}
\operatorname{deg} u_{1}+\operatorname{deg} v_{1}+\operatorname{deg} u_{2} & \leq n-|V(H)|+\operatorname{deg}_{H} u_{1}+\operatorname{deg}_{H} v_{1}+\operatorname{deg}_{H} u_{2} \\
& \leq n-5+1+3+2=n+1 .
\end{aligned}
$$

Suppose $\operatorname{deg} u_{1}+\operatorname{deg} v_{1}+\operatorname{deg} u_{2}=n+1$. Then, putting $U_{1}=N\left(u_{1}\right) \cap$ $V(C), U_{2}=N\left(u_{2}\right) \cap V(C)$ and $V_{1}=N\left(v_{1}\right) \cap V(C)-\left\{u_{1}, u_{2}\right\}$, we have $U_{1} \neq$ $\emptyset \neq U_{2}$ and each vertex of $C-\left\{u_{1}, u_{2}\right\}$ is in exactly one of the sets $U_{1}, U_{2}$ and $V_{1}$. Since $C$ is connected, there exists an edge $u v$ of $C$ with $u \in U_{1}$ and $v \in U_{2} \cup V_{1}$. If $v \in V_{1}$, then Case 1 applies to the neighbors $u_{1}$ and $v$ of $K$ on $C$, contrary to assumption. If $v \in U_{2}$, then Case 2 applies to $u_{1}$ and $u_{2}$, again contrary to assumption. We conclude that

$$
\operatorname{deg} u_{1}+\operatorname{deg} v_{1}+\operatorname{deg} u_{2} \leq n \text {. }
$$

By Lemma 2, $N\left(v_{1}\right) \cap N(w) \cap(V(G)-V(C))=N\left(u_{1}\right) \cap N(w) \cap(V(G)-$ $V(C))=\emptyset$. Assuming that $N\left(v_{1}\right) \cap N(w) \cap V(C)-\left\{u_{2}\right\} \neq \emptyset$ or $N\left(u_{1}\right) \cap$ $N(w) \cap V(C) \neq \emptyset$, we reach the contradiction that Case 1 or Case 2 applies. Hence

$$
N\left(v_{1}\right) \cap N(w) \cap(V(G)-V(H))=N\left(u_{1}\right) \cap N(w) \cap(V(G)-V(H))=\emptyset .
$$

Together with (12) we obtain

$$
\begin{aligned}
\operatorname{deg} u_{1}+\operatorname{deg} v_{1}+\operatorname{deg} w & \leq n-|V(H)|+\operatorname{deg}_{H} u_{1}+\operatorname{deg}_{H} v_{1}+\operatorname{deg}_{H} w \\
& \leq n-5+1+3+1=n
\end{aligned}
$$

Summation of (13) and (15) yields

$$
2\left(\operatorname{deg} u_{1}+\operatorname{deg} v_{1}\right)+\operatorname{deg} u_{2}+\operatorname{deg} w \leq 2 n,
$$

so that either $\operatorname{deg} u_{1}+\operatorname{deg} v_{1} \leq \frac{2}{3} n$ or $\operatorname{deg} u_{2}+\operatorname{deg} w \leq \frac{2}{3} n$.

Corollary 5. Let $G$ be a connected, almost bridgeless graph of order $n \geq 4$ such that $\operatorname{deg} u+\operatorname{deg} v \geq(2 n+1) / 3$ for every edge $u v$ of $G$. Then $L(G)$ is hamiltonian. Moreover, if $G \not C_{4}, C_{5}$, then $L(G)$ is pancyclic. 
Proof. Let $G$ be a connected, almost bridgeless graph of order $n \geq 4$ such that $\operatorname{deg} u+\operatorname{deg} v \geq(2 n+1) / 3$ for every edge $u v$ of $G$. The existence of a hamiltonian cycle in $L(G)$ immediately follows from the combination of Theorems 1 and 4 . If $G \cong K_{1, n-1}$, then $L(G)$ is complete and hence pancyclic. Now assume $G \not \equiv C_{4}, C_{5}, K_{1, n-1}$ and $L(G)$ is not pancyclic. Let $k=\max \{i \mid L(G)$ does not contain $\left.C_{i}\right\}$.

We have $\Delta(G) \geq 3$, so $k \geq 4$. Let $D=u_{1} u_{2} \ldots u_{p} u_{1}$ be a shortest cycle in $G$ and suppose $p \geq 5$. Then every vertex of $G-V(D)$ is adjacent to at most one vertex of $D$, implying that

$$
p(2 n+1) / 6 \leq \sum_{i=1}^{p} \operatorname{deg} u_{i} \leq n-p+2 p,
$$

so that $n \leq\lfloor 5 p /(2 p-6)\rfloor \leq 6$. However, it is easily checked that every graph of order at most 6 satisfying our assumptions has a cycle of length at most 4 . Hence, in fact, $p \leq 4$ and

$$
\begin{aligned}
\beta(D) & \geq p+\sum_{i=1}^{p}\left(\operatorname{deg} u_{i}-2\right) \geq\lceil-p+p(2 n+1) / 6\rceil \\
& =\lceil p(2 n-5) / 6\rceil \geq\lceil(2 n-5) / 2\rceil=n-2 .
\end{aligned}
$$

Observing that, for any circuit $C$ of $G, L(G)$ contains a cycle of length $i$ for every $i$ with $|E(C)| \leq i \leq \beta(C)$, we conclude that $k \geq n-1$.

$L(G)$ is hamiltonian, so $k<|E(G)|$ and $L(G)$ contains $C_{k+1}$. Hence $G$ contains a circuit $C$ with $|E(C)| \leq k+1 \leq \beta(C)$. In fact $|E(C)|=k+1$, otherwise $L(G)$ contains $C_{k}$. Since $C$ is a circuit, there exists edge-disjoint cycles $D_{1}, D_{2}, \ldots, D_{r}$ such that $C=\cup_{i=1}^{r} D_{i}$. We now derive contradictions in two cases.

Case 1. $r=1$.

Since $|E(C)|=k+1 \geq n, C$ is a hamiltonian cycle of $G$ and $k=n-1$. Let $D^{\prime}$ be a shortest cycle among all cycles of $G$ that contain exactly one chord of $C$. Let $D^{\prime}$ have length $q$. If $q=3$, then $G$, and hence $L(G)$ too, contains $C_{n-1}$, a contradiction. If $q \geq 4$, then $n \geq 6$ and as in (16) we obtain

$$
\beta\left(D^{\prime}\right) \geq\lceil q(2 n-5) / 6\rceil \geq\lceil 4(2 n-5) / 6\rceil \geq n-1,
$$

again implying the contradiction that $L(G)$ contains $C_{n-1}$.

Case 2. $r \geq 2$.

Let $H$ be the graph with $V(H)=\left\{D_{1}, D_{2}, \ldots, D_{r}\right\}$ and $D_{i} D_{j} \in E(H)$ if and only if $V\left(D_{i}\right) \cap V\left(D_{j}\right) \neq \emptyset$. Since $H$ is connected, at least two vertices of $H$ are not cut vertices of $H$. Equivalently, there are at least two values of $j$ for which $\cup_{1 \leq i \leq r, i \neq j} D_{i}$ is a connected subgraph of $G$ and hence a circuit of $G$. Assume 
without loss of generality that $C^{\prime}=\cup_{i=2}^{r} D_{i}$ and $C^{\prime \prime}=D_{1} \cup \cup_{i-3}^{r} D_{i}$ are circuits of $G$. If $E\left(D_{2}-V\left(C^{\prime \prime}\right)\right)=\emptyset$, then $\left|E\left(C^{\prime \prime}\right)\right|<|E(C)|=k+1 \leq \beta\left(C^{\prime \prime}\right)$, so that $L(G)$ contains $C_{k}$. Hence there exists an edge $u v$ of $D_{2}$ with $u, v \notin$ $V\left(C^{\prime \prime}\right)$. Let $E_{1}$ be the set of edges of $D_{1}$ incident with at least one vertex of $C^{\prime}$ and $E_{2}=E\left(D_{1}\right)-E_{1}$. Then

$$
\begin{aligned}
\beta\left(C^{\prime}\right) \geq & \left|E\left(C^{\prime}\right)\right|+\left|E_{1}\right|+\operatorname{deg} u-2+\operatorname{deg} v-2 \geq|E(C)|-\left|E_{2}\right| \\
& +(2 n+1) / 3-4 .
\end{aligned}
$$

On the other hand, since $L(G)$ does not contain $C_{k}$,

$$
\beta\left(C^{\prime}\right) \leq k-1=|E(C)|-2 .
$$

It follows that $\left|E_{2}\right| \geq(2 n-5) / 3$. Hence $\left|V\left(D_{1}-V\left(C^{\prime}\right)\right)\right| \geq(2 n-2) / 3$ and similarily $\left|V\left(D_{2}-V\left(C^{\prime \prime}\right)\right)\right| \geq(2 n-2) / 3$. But then

$$
\begin{aligned}
n=|V(G)| & \geq\left|V\left(D_{1}-V\left(C^{\prime}\right)\right)\right|+\left|V\left(D_{2}-V\left(C^{\prime \prime}\right)\right)\right|+1 \\
& \geq 2(2 n-2) / 3+1>n,
\end{aligned}
$$

a contradiction.

We do not know any connected, almost bridgeless graph $G$ of order $n$ without a $D$-circuit such that $G \not \equiv K_{\mathrm{I}, n-1}$ and $\operatorname{deg} u+\operatorname{deg} v \geq \frac{2}{3} n$ for every edge $u v$ of $G$. We conjecture that, for $n$ sufficiently large, the bound $(2 n+1) / 3$ in Theorem 4 and Corollary 5 can be decreased to $(2 n-9) / 5$. If true, this conjecture is best possible. To see this, construct for $i \geq 3$ a graph $G(i)$ as follows: take five disjoint copies of $K_{i}$, label them $G_{1}, \ldots, G_{5}$; choose three vertices $u_{1}$, $u_{2}, u_{3}$ in $G_{1}$, three vertices $v_{1}, v_{2}, v_{3}$ in $G_{2}$, two vertices $x_{1}, x_{2}$ in $G_{3}$, two vertices $y_{1}, y_{2}$ in $G_{4}$ and two vertices $z_{1}, z_{2}$ in $G_{5}$; obtain $G(i)$ as $\cup_{j-1}^{5} G_{j}+$ $\left\{u_{1} x_{1}, u_{2} y_{1}, u_{3} z_{1}, v_{1} x_{2}, v_{2} y_{2}, v_{3} z_{2}\right\}$. Then $G(i)$ is 2 -connected and $\operatorname{deg} u+$ $\operatorname{deg} v \geq(2|V(G(i))|-10) / 5$ for every edge $u v$ of $G(i)$, while $G(i)$ contains no $D$-circuit and hence $L(G(i))$ is nonhamiltonian.

Although Corollary 5 may not be best possible, it is strong enough to contain Clark's result.

Corollary 6. (Clark [3]). Let $G$ be a connected graph of order $n \geq 6$. If $\operatorname{deg} u+\operatorname{deg} v \geq n-1-p(n)$ for every edge $u v$ of $G$, where $p(n)=0$ if $n$ is even and $p(n)=1$ if $n$ is odd, then $L(G)$ is hamiltonian.

Proof. Let $G$ be a connected graph of order $n \geq 6$ such that deg $u+$ $\operatorname{deg} v \geq n-1-p(n)$ for every edge $u v$ of $G$. Since $n \geq 6, n-1-$ $p(n) \geq(2 n+1) / 3$. Hence we are done by Corollary 5 if $G$ is shown to be almost bridgeless. Suppose $G$ contains a bridge $u_{1} u_{2}$ with $\operatorname{deg} u_{1} \neq 1 \neq$ 
deg $u_{2}$. Let $H_{i}$ be the component of $G-u_{1} u_{2}$ containing $u_{i}(i=1,2)$. Assume without loss of generality that $\left|V\left(H_{1}\right)\right| \leq\left|V\left(H_{2}\right)\right|$, so that $\left|V\left(H_{1}\right)\right| \leq(n-p(n)) /$ 2. Since $\left|V\left(H_{1}\right)\right| \geq 2, H_{1}-u_{1}$ contains a vertex $u$. If $u$ has a neighbor $v$ with $v \neq u_{1}$, then $\operatorname{deg} u+\operatorname{deg} v \leq 2\left(\left|V\left(H_{1}\right)\right|-1\right) \leq n-p(n)-2$, a contradiction. If $u$ has no neighbor in $H-u_{1}$, then $u u_{1} \in E(G)$ and deg $u=1$, so that $\operatorname{deg} u+\operatorname{deg} u_{1} \leq 1+\left|V\left(H_{1}\right)\right| \leq 1+(n-p(n)) / 2$. For $n \geq 6$ we have $1+(n-p(n)) / 2 \leq n-2-p(n)$. Thus $\operatorname{deg} u+\operatorname{deg} u_{1} \leq n-2-p(n)$, again a contradiction.

The bound $(2 n+1) / 3$ in Corollary 5 can be decreased in case only hamiltonian graphs are considered.

Theorem 7. Let $G$ be a hamiltonian graph of order $n \geq 13$. If $\operatorname{deg} u+$ deg $v \geq n / 2$ for every edge $u v$ of $G$, then $L(G)$ is pancyclic.

For the proof of Theorem 7 we refer to [1].

\section{SPANNING CIRCUITS}

In [6] Lesniak-Foster and Williamson proved that a graph $G$ contains an $S$ circuit if $|V(G)|=n \geq 6, \delta(G) \geq 2$ and $\operatorname{deg} u+\operatorname{deg} v \geq n-1$ for every pair of nonadjacent vertices $u$ and $v$. All graphs showing that this resuit is best possible contain a bridge. For a graph $G$ to have an $S$-circuit it is necessary that $G$ is connected and contains no bridges. We now show how the above result can be improved by additionally imposing these necessary conditions.

Theorem 8. Let $G$ be a connected bridgelsss graph of order $n \geq 3$. If $\operatorname{deg} u+\operatorname{deg} v \geq(2 n+3) / 3$ for every pair of nonadjacent vertices $u$ and $v$, then $G$ contains an S-circuit.

Proof. Let $G$ be a connected bridgeless graph of order $n \geq 3$. Assuming that $G$ contains no $S$-circuit, we will exhibit two nonadjacent vertices with degree-sum smaller than $(2 n+3) / 3$. Since $G$ is bridgeless, $G$ contains a circuit. Let $C$ be a circuit of $G$ of maximum order and $K$ a component of $G-V(C)$. By Lemma 2 and the fact that $G$ is bridgeless, $K$ has at least two neighbors on $C$. We distinguish three cases.

Case 1. $K$ has two neighbors on $C$ which are joined by a path of length 2 contained in $G-V(K)$.

Let $u_{1}$ and $u_{2}$ be two neighbors of $K$ on $C$ which are joined by the path $u_{1} w_{1} u_{2}$, where $w_{1} \notin V(K)$. Let $P$ be a $u_{1}-u_{2}$ path with $\emptyset \neq V(P)-\left\{u_{1}, u_{2}\right\} \subset$ $V(K)$ such that $|V(P)|$ is minimum and let $v$ be an arbitrary vertex in $V(P) \cap$ $V(K)$. We distinguish two subcases. 
Case 1.1. $u_{1}$ and $u_{2}$ have a common neighbor $w_{2} \in V(G)-\left(V(K) \cup\left\{w_{1}\right\}\right)$.

Put $H=\left\langle V(P) \cup\left\{w_{1}, w_{2}\right\}\right\rangle$. Lemmas 2, 3(d) and 3(e) imply that $\left\{v, w_{1}, w_{2}\right\}$ is an independent set and each vertex of $G-V(H)$ is adjacent to at most one of the vertices $v, w_{1}$, and $w_{2}$. Together with the minimality of $|V(P)|$ we obtain

$$
\begin{aligned}
\operatorname{deg} v+\operatorname{deg} w_{1}+\operatorname{deg} w_{2} & \leq n-|V(H)|+\operatorname{deg}_{H} v+\operatorname{deg}_{H} w_{1}+\operatorname{deg}_{H} w_{2} \\
& \leq n-5+2+2+2=n+1 .
\end{aligned}
$$

It follows that at least one of the nonadjacent vertex pairs $\left\{v, w_{1}\right\},\left\{v, w_{2}\right\}$ and $\left\{w_{1}, w_{2}\right\}$ has degree-sum at most $2(n+1) / 3$, settling Case 1.1 .

Case 1.2. $u_{1}$ and $u_{2}$ have no common neighbor in $V(G)-\left(V(K) \cup\left\{w_{1}\right\}\right)$.

Put $H=\left\langle V(P) \cup\left\{w_{1}\right\}\right\rangle$. By Lemmas 2, 3(b) and 3(d), each vertex of $G-$ $V(H)$ is adjacent to at most one of the vertices $u_{1}, u_{2}, v, w_{1}$, so that

$$
\begin{gathered}
\operatorname{deg} u_{1}+\operatorname{deg} u_{2}+\operatorname{deg} v+\operatorname{deg} w_{1} \leq n-|V(H)|+\operatorname{deg}_{H} u_{1}+\operatorname{deg}_{H} u_{2} \\
+\operatorname{deg}_{H} v+\operatorname{deg}_{H} w_{1} \leq n-4+2+2+2+2=n+4 .
\end{gathered}
$$

It follows that at least one of the nonadjacent vertex pairs $\left\{u_{1}, u_{2}\right\}$ and $\left\{v, w_{1}\right\}$ has degree-sum at most $(n+4) / 2$. If $n>6$, then $(n+4) / 2<(2 n+3) / 3$ and we are done. Now assume $n \leq 6$. Since $\operatorname{deg}_{C} u_{i} \geq 2, u_{i}$ has a neighbor $v_{i}$ on $C$ with $v_{i} \neq w_{1}(i=1,2)$. By assumption $v_{1}$ and $v_{2}$ do not coincide, so that $n \geq 6$ and hence $n=6$. By Lemmas 2 and $3(\mathrm{~b}),\left(N(v) \cup N\left(w_{1}\right)\right) \cap$ $\left\{v_{1}, v_{2}\right\}=\emptyset$. Thus $\operatorname{deg} v=\operatorname{deg} w_{1}=2$, so that $\operatorname{deg} v+\operatorname{deg} w_{1}=4<5=$ $(2 n+3) / 3$.

Case 2. Case 1 does not apply and $\mathrm{K}$ has two neighbors on $C$ which are joined by a path of length 3 contained in $G-V(K)$.

Let $u_{1}$ and $u_{2}$ be two neighbors of $K$ on $C$ which are joined by the path $u_{1} w_{1} w_{2} u_{2}$, where $w_{1}, w_{2} \notin V(K)$. Define $P$ and $v$ as in Case 1 and put $H=$ $\left\langle V(P) \cup\left\{w_{1}, w_{2}\right\}\right\rangle$. By Lemma 3(c) at least one of the following three subcases applies.

Case 2.1. $\quad N\left(u_{1}\right) \cap N\left(w_{1}\right)=N\left(u_{2}\right) \cap N\left(w_{2}\right)=\emptyset$.

By Lemma 2 and the fact that Case 1 does not apply, each vertex of $G-V(H)$ is adjacent to at most one of the vertices $u_{1}, v$ and $w_{1}$. Hence

$$
\begin{aligned}
\operatorname{deg} u_{1}+\operatorname{deg} v+\operatorname{deg} w_{1} & \leq n-|V(H)|+\operatorname{deg}_{H} u_{1}+\operatorname{deg}_{H} v+\operatorname{deg}_{H} w_{1} \\
& \leq n-5+2+2+2=n+1
\end{aligned}
$$

Similarly,

$$
\operatorname{deg} u_{2}+\operatorname{deg} v+\operatorname{deg} w_{2} \leq n+1
$$


Assuming without loss of generality that $\operatorname{deg} w_{1} \leq \operatorname{deg} w_{2}$ we deduce from (17) and (18) that

$$
\begin{aligned}
2\left(\operatorname{deg} v+\operatorname{deg} w_{1}\right)+\operatorname{deg} u_{1}+\operatorname{deg} u_{2} \leq & 2 \operatorname{deg} v+\operatorname{deg} w_{1}+\operatorname{deg} w_{2} \\
& +\operatorname{deg} u_{1}+\operatorname{deg} u_{2} \leq 2 n+2 .
\end{aligned}
$$

Hence one of the nonadjacent vertex pairs $\left\{v, w_{1}\right\}$ and $\left\{u_{1}, u_{2}\right\}$ has degree-sum at most $(2 n+2) / 3$.

Case 2.2 $N\left(u_{1}\right) \cap N\left(w_{1}\right)=N\left(w_{1}\right) \cap N\left(w_{2}\right)=\emptyset$.

Similar arguments as used in Case 2.1 now yield

$$
\operatorname{deg} u_{1}+\operatorname{deg} v+\operatorname{deg} w_{1} \leq n+1
$$

and

$$
\operatorname{deg} v+\operatorname{deg} w_{1}+\operatorname{deg} w_{2} \leq n+1 \text {, }
$$

implying that

$$
2\left(\operatorname{deg} v+\operatorname{deg} w_{1}\right)+\operatorname{deg} u_{1}+\operatorname{deg} w_{2} \leq 2 n+2 .
$$

Hence either $\operatorname{deg} v+\operatorname{deg} w_{1} \leq(2 n+2) / 3$ or $\operatorname{deg} u_{1}+\operatorname{deg} w_{2} \leq(2 n+2) / 3$.

Case 2.3. $\quad N\left(u_{2}\right) \cap N\left(w_{2}\right)=N\left(w_{1}\right) \cap N\left(w_{2}\right)=\emptyset$.

This case is symmetric to Case 2.2 .

Case 3. Neither Case 1 nor Case 2 applies.

Let $u_{1}$ and $u_{2}$ be two neighbors of $K$ on $C$ and, for $i=1,2, w_{i}$ a vertex in $N\left(u_{i}\right)-V(K)$. Define $P$ and $v$ as in Case 1 and put $H=\left\langle V(P) \cup\left\{w_{1}, w_{2}\right\}\right\rangle$. By Lemma 2 and the fact that neither Case 1 nor Case 2 applies, each vertex of $G-V(H)$ is adjacent to at most one of the vertices $u_{1}, v$ and $w_{2}$. Hence

$$
\begin{aligned}
\operatorname{deg} u_{1}+\operatorname{deg} v+\operatorname{deg} w_{2} & \leq n-|V(H)|+\operatorname{deg}_{H} u_{1}+\operatorname{deg}_{H} v+\operatorname{deg}_{H} w_{2} \\
& \leq n-5+2+2+1=n .
\end{aligned}
$$

Similarly,

$$
\operatorname{deg} u_{2}+\operatorname{deg} v+\operatorname{deg} w_{1} \leq n .
$$

Assuming without loss of generality that $\operatorname{deg} w_{1} \leq \operatorname{deg} w_{2}$, we obtain

$$
2\left(\operatorname{deg} v+\operatorname{deg} w_{1}\right)+\operatorname{deg} u_{1}+\operatorname{deg} u_{2} \leq 2 n .
$$

Hence either $\operatorname{deg} v+\operatorname{deg} w_{1} \leq \frac{2}{3} n$ or $\operatorname{deg} u_{1}+\operatorname{deg} u_{2} \leq \frac{2}{3} n$. 
The graph $K_{2,3}$ is the only known example of a connected bridgeless graph of order $n \geq 3$ without an $S$-circuit such that $\operatorname{deg} u+\operatorname{deg} v \geq(2 n+2) / 3$ for every pair of nonadjacent vertices $u$ and $v$. We conjecture that the bound in Theorem 8 , too, can be decreased to $(2 n-9) / 5$ if $n$ is sufficiently large. Such an improvement would be best possible in view of the graphs $G(i)$ defined in Section 2.

Theorem 8 implies the result of Lesniak-Foster and Williamson mentioned above.

Corollary 9. (Lesniak-Foster and Williamson [6]). Let $G$ be a graph with $|V(G)|=n \geq 6$ and $\delta(G) \geq 2$. If $\operatorname{deg} u+\operatorname{deg} v \geq n-1$ for every pair of nonadjacent vertices $u$ and $v$, then $G$ contains an $S$-circuit.

Proof. Let $G$ be a graph with $|V(G)|=n \geq 6$ and $\delta(G) \geq 2$ such that $\operatorname{deg} u+\operatorname{deg} v \geq n-1$ for every pair of nonadjacent vertices $u$ and $v$. It is easily seen that $G$ must be connected. Since $n \geq 6, n-1 \geq(2 n+3) / 3$. In view of Theorem 8 it remains to be shown that $G$ is bridgeless. Suppose $G$ contains a bridge $u_{1} u_{2}$. Let $H_{i}$ be the component of $G-u_{1} u_{2}$ containing $u_{i}$ $(i=1,2)$. Since $\delta(G) \geq 2, H_{i}$ is nontrivial, say that $v_{i} \in V\left(H_{i}\right)-\left\{u_{i}\right\}$ $(i=1,2)$. Then $v_{1} v_{2} \notin E(G)$ and $\operatorname{deg} v_{1}+\operatorname{deg} v_{2} \leq\left|V\left(H_{1}\right)\right|-1+$ $\left|V\left(H_{2}\right)\right|-1=n-2$, a contradiction.

\section{DOMINATING CIRCUITS REVISITED}

A slight variation on the proof of Theorem 8 gives us the following counterpart of Theorem 4.

Theorem 10. Let $G$ be a connected, almost bridgeless graph of order $n \geq 3$. If $\operatorname{deg} u+\operatorname{deg} v \geq(2 n+1) / 3$ for every pair of nonadjacent vertices $u$ and $v$, then $G$ contains a $D$-circuit.

Proof outline. Let $G$ be a connected, almost bridgeless graph of order $n \geq 3$. We will exhibit a nonadjacent vertex pair with degree-sum smaller than $(2 n+1) / 3$ under the assumption that $G$ contains no $D$-circuit. Let $C$ be a circuit of $G$ of maximum order and $K$ a nontrivial component of $G-V(C) . K$ has at least two neighbors on $C$.

Distinguish the same cases as in the proof of Theorem 8 . In each case define $P$ as a shortest $u_{1}-u_{2}$ path with $\emptyset \neq V(P)-\left\{u_{1}, u_{2}\right\} \subset V(K)$ and $v_{1}$ as the successor of $u_{1}$ on $P$. If $V(P)-\left\{u_{1}, u_{2}\right\}=\left\{v_{1}\right\}$, let $v$ be an arbitrary neighbor of $v_{1}$ in $K$, otherwise let $v$ be the successor of $v_{1}$ on $P$. Now all upper bounds on degree-sums in the proof of Theorem 8 can be decreased to obtain a vertex pair as desired.

Without proof we mention that the corresponding counterpart of Corollary 5 also holds. 
Corollary 11. Let $G$ be a connected, almost bridgeless graph of order $n \geq 3$ such that $\operatorname{deg} u+\operatorname{deg} v \geq(2 n+1) / 3$ for every pair of nonadjacent vertices $u$ and $v$. Then $L(G)$ is hamiltonian. Moreover, if $G \neq C_{4}, C_{5}$, then $L(G)$ is pancyclic.

Again we conjecture, as a best possible improvement of Theorem 10 and Corollary 11 , that the bound $(2 n+1) / 3$ can be decreased to $(2 n-9) / 5$ for $n$ sufficiently large.

Note added in proof. A graph $G$ is cyclically 2-edge-connected if no two cycles of $G$ can be separated by the removal of at most one edge. Suppose $G$ has order $n \geq 5$ with deg $u+\operatorname{deg} v \geq(2 n+1) / 3$ for every edge $u v$ of $G$. Then $G$ is connected and almost bridgeless if and only if $G$ is cyclically 2-edge-connected and has no isolated vertices. Consequently, a corollary of Theorem 4 is the following: Let $G$ be a nontrivial cyclically 2-edge-connected graph of order $n$ with no isolated vertices. If $\operatorname{deg} u+\operatorname{deg} v \geq(2 n+1) / 3$ for every edge $u v$ of $G$, then $G$ contains a $D$-circuit. Here the bound $(2 n+1) / 3$ is best possible, as the following example shows. Let $u$ be any vertex in $K_{\left(n^{n} ; \mid\right)-1}, v$ the center of the $\operatorname{star} K_{1,(2 n / 3)-2}$ and $G=\left(K_{(n / 3)-1} \cup K_{1,(2 n / 3)-2}\right)+u v$. Then $G$ satisfies the above conditions with $(2 n+1) / 3$ replaced with $2 n / 3$ but $G$ has no $D$-circuit, since $L(G)$ is not hamiltonian.

\section{References}

[1] A. Benhocine, Problèmes hamiltoniens et pancyclisme dans les graphes adjoints. Thesis, Paris XI, France (1986).

[2] R. A. Brualdi and R. F. Shanny, Hamiltonian line graphs. J. Graph Theory 5 (1981) 307-314.

[3] L. Clark, On hamiltonian line graphs. J. Graph Theory 8 (1984) 303-307.

[4] F. Harary, Graph Theory. Addison-Wesley, Reading, Mass. (1969).

[5] F. Harary and C. St. J. A. Nash-Williams, On eulerian and hamiltonian graphs and line graphs. Canad. Math. Bull. 8 (1965) 701-710.

[6] L. Lesniak-Foster and J. E. Williamson, On spanning and dominating circuits in graphs. Canad. Math. Bull. 20 (1977) 215-220.

[7] H. J. Veldman, Existence of spanning and dominating trails and circuits. To appear in J. Graph Theory. 